\title{
Muscle weakness after rest in myotonic disorders : an electrophysiological study
}

\author{
J. COLIN BROWN \\ From the Department of Neurology, Royal Victoria Infirmary, Newcastle Upon Tyne
}

SYNOPSIS Changes in amplitude of the evoked muscle action potential (MAP) have been observed in four patients with myotonia congenita and two with dystrophia myotonica. A fade in the response occurred in every case with stimulus frequencies of 10 per second or less, provided that the muscle was in a rested state and that long enough stimulus trains were used. Intramuscular stimulation and recording techniques show that the myotonic muscle fibre is the site of this defect. The MAP fade is thought to represent the transient weakness from which such patients may suffer, particularly after rest. Since neither this weakness nor the fade was related to the severity of the myotonia, nor were they significantly influenced by cooling or hydantoins, they may well be due to a separate defect ino the myotonic muscle from that which causes the hyperexcitability of the fibre membrane.

Alterations in the amplitude of both mechanical and electrical muscle responses evoked by repetitive nerve stimulation have been noted in myotonic disorders (Lambert and Beckett, 1950; McComas et al., 1971; Ricker et al., 1973). Kirby and Kraft (1973) have suggested that these changes might be related to the transient weakness which may occur in patients with myotonia. However, when observed, these changes have usually been transitory, inconstant, or evident in only a proportion of subjects studied. A study of the effect of repetitive nerve stimulation upon the evoked muscle action potential (MAP) was therefore undertaken in patients with myotonia congenita and dystrophia myotonica in an attempt to define the extent and degree of these changes and the site of the defect responsible for them.

\section{METHODS}

Four patients with myotonia congenita (three being siblings) and two with dystrophia myotonica were studied (Table). Those with myotonia congenita had noticed muscle stiffness throughout their lives. Those with dystrophia myotonica had noticed 1 Present address: Norfolk and Norwich Hospital, Norwich, Norfolk,
NR1 3SR.
INTERVAL SINCE PREVIOUS TRAIN
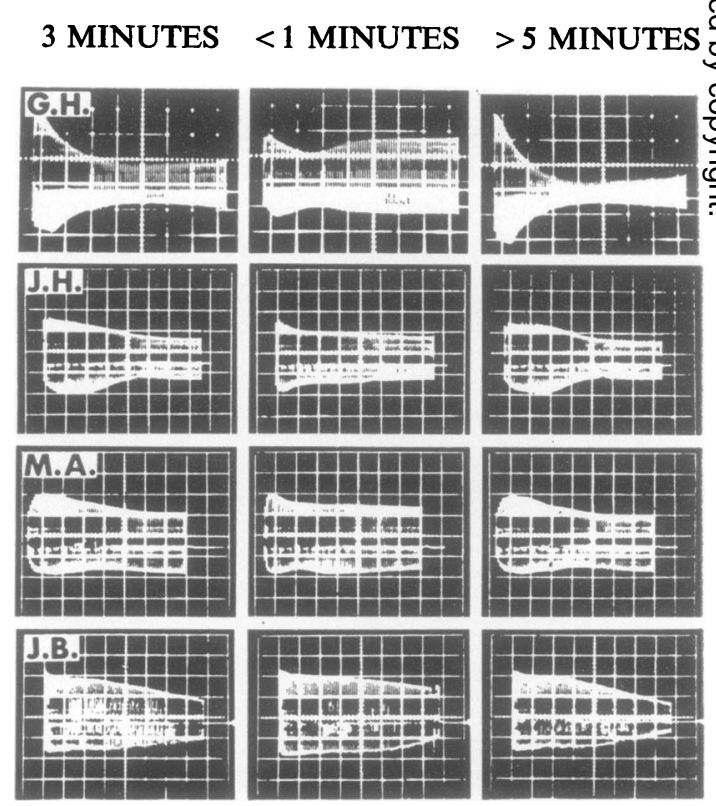

FIG. 1. MAP responses to eight second stimulus trains at 10 per second. Note that the fade is less when recorded soon after the previous train and greater after more than five minutes. Vertical scale; G.H. and J.H. $-5 \mathrm{mV}$ per division. M.A. and J.B. $-2 \mathrm{mV}$ per division. Horizontal scale; one second per division. 
TABLE

DETAILS OF PATIENTS STUDIED

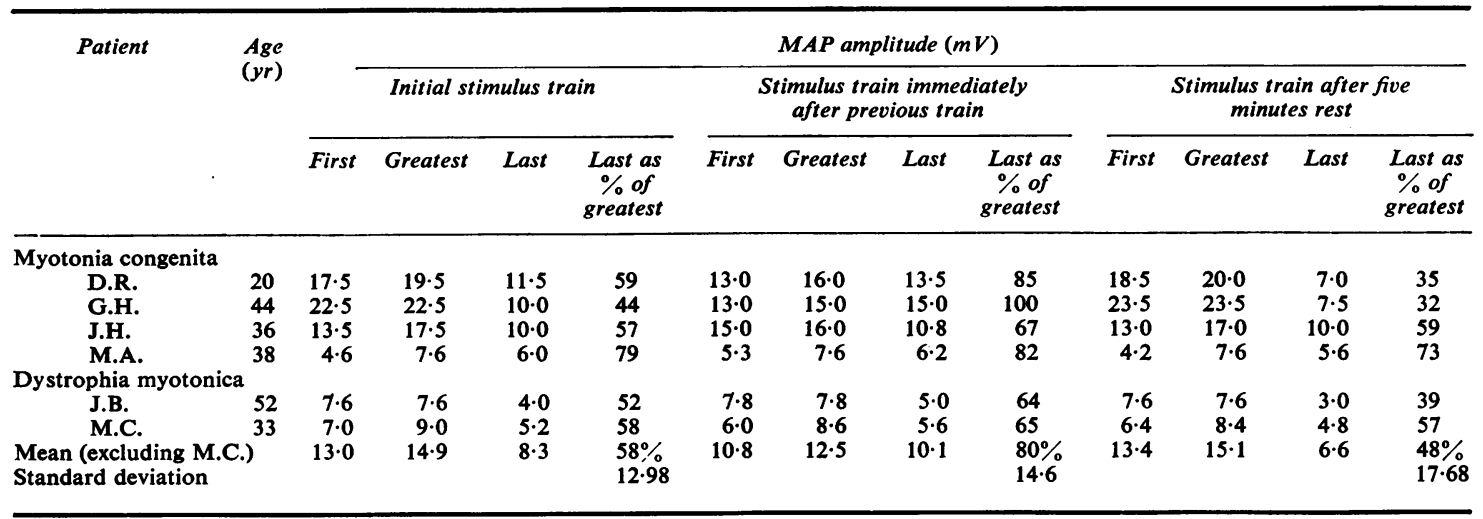

Stimulus frequencies, 10 per second in all subjects. Duration of stimulus train, eight seconds in all except M.C. in whom 20 second train used.

D.R.

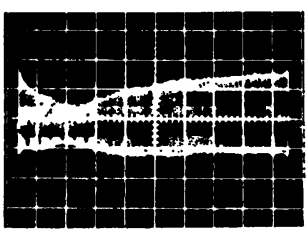

J.H.

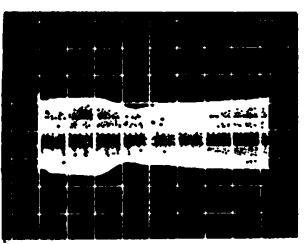

J.B.

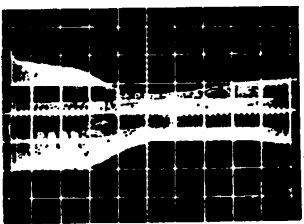

FIG. 2. MAP responses to 40-50 second stimulus trains at seven per second. The 'decline-rise' is prominent in D.R. and G.H. and just apparent in J.H. and M.A. The 'rise' is only slight in J.B. Vertical scale: D.R., G.H., and J.H. $-5 \mathrm{mV}$ per division. M.A. and J.B. $-2 \mathrm{mV}$ per division. Horizontal scale; five seconds per division.

\section{G.H.}

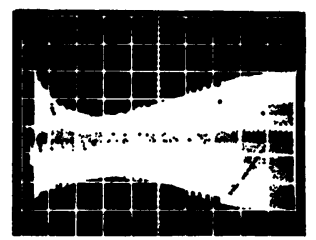

M.A.
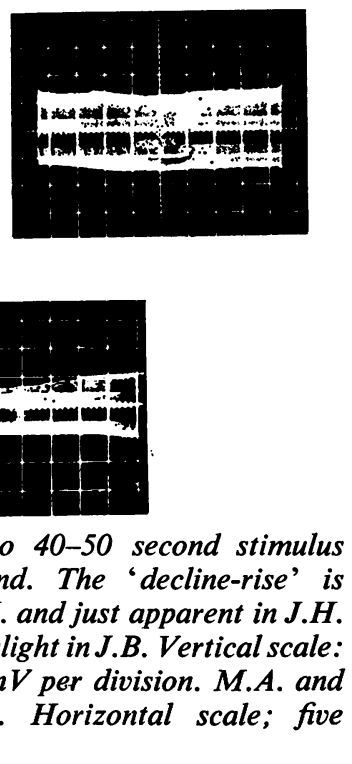
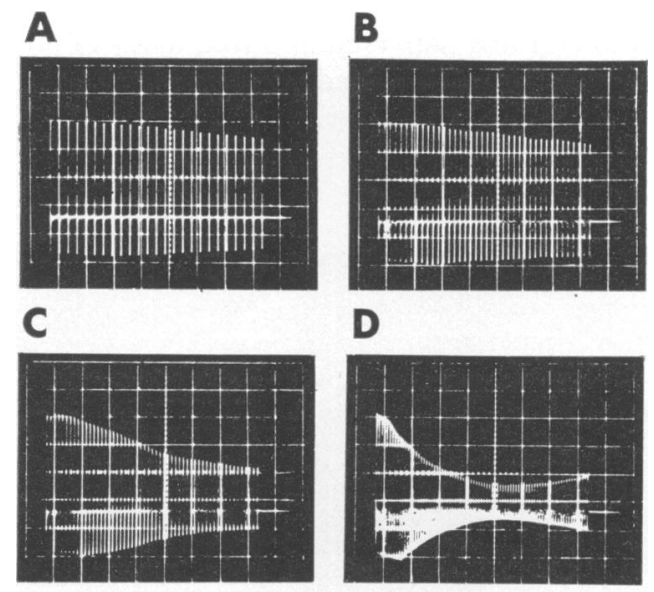

D
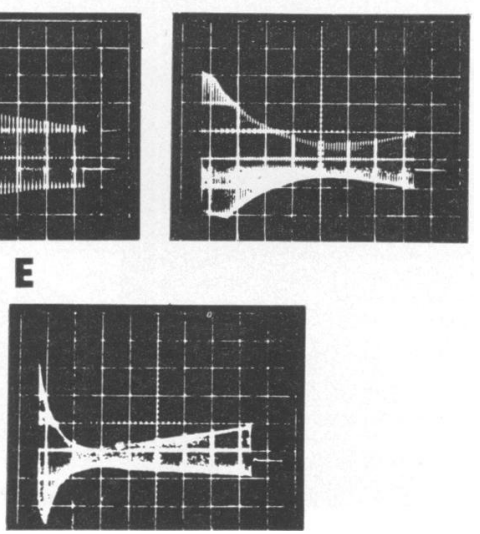

FIG. 3. MAP responses at different stimulus frequencies in G.H. A, three per second, $\mathrm{B}$, five per second, $\mathrm{C}$, seven per second, D, eight per second, $\mathrm{E}$, 20 per second. Vertical scale: $5 \mathrm{mV}$ per division. Horizontal scale: one second per division. 
weakness and stiffness for between 10 and 15 years. All medication was stopped at least 24 hours before each study.

The technique was similar to that described by Grob et al. (1956). The ambient temperature was regulated between $20-22^{\circ} \mathrm{C}$. Supramaximal stimuli (the intensity being $150 \%$ of that required to evoke the entire MAP) of $0.2 \mathrm{~ms}$ duration were delivered to the ulnar nerve at the wrist through surface electrodes from a Devices stimulator (type 3072) triggered by a digitimer through a gated pulse generator.

Recordings of the evoked MAP from the abductor digiti minimi were made between surface electrodes of $10 \mathrm{~mm}$ diameter, one situated over the belly of the muscle and the other as a reference over the base of the fifth finger. The potentials were amplified through Medelec parametric (type 2A3) and Tektronix (type 3A74) amplifiers and displayed on a storage oscilloscope. Earthing was effected by an electrode encircling the wrist distal to the stimulating electrodes. Isometric contraction was ensured by mechanical fixation of the hand to a board.

\section{RESULTS}

In the first patient studied (D.R.), eight second stimulus trains delivered at a frequency of 10 per second resulted in a fade of the evoked MAP. However, this fade varied considerably on

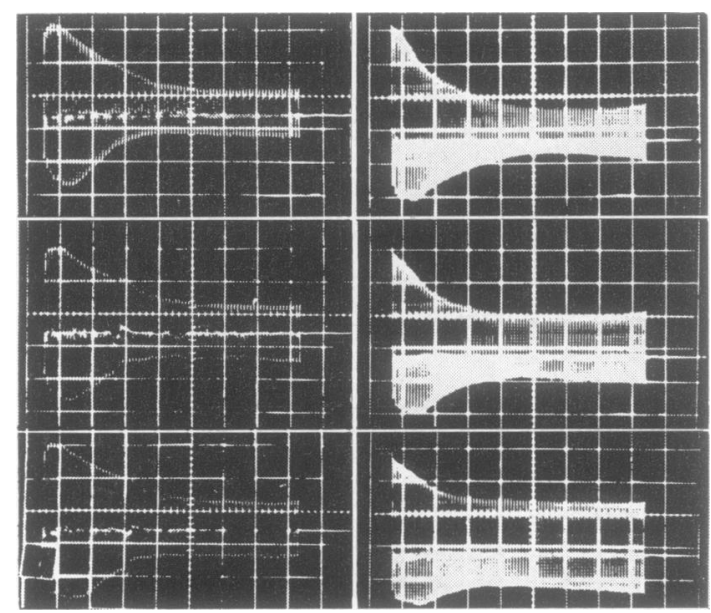

FIG. 5. Effect of $10 \mathrm{mg}$ edrophonium upon MAP responses in D.R. (right) and G.H. (left). Top: before. Centre: one minute after. Bottom: four minutes after injection. Vertical scale, $5 \mathrm{mV}$ per division. Horizontal scale, one second per division.

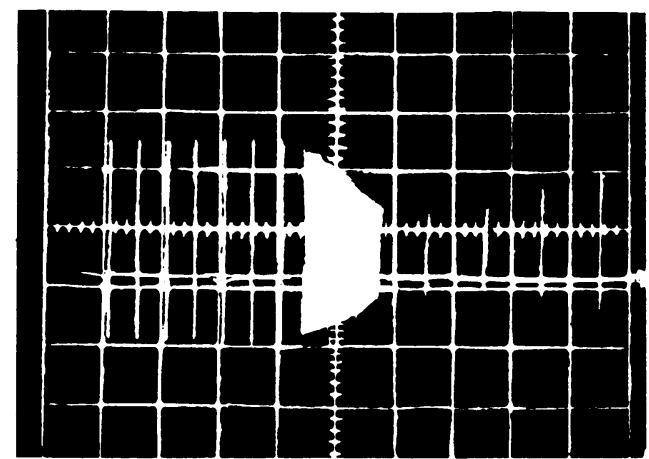

FIG. 4. Post-tetanic depression of MAP response in J.B. One per second nerve stimulation has been interupted by a two second stimulus train at 30 per second. Vertical scale; $2 \mathrm{mV}$ per division. Horizontal scale; one second per division.

different occasions. It became evident that this depended upon whether a stimulus train wasis delivered after a period of rest or soon after theo previous train. If the recording were made्ञ immediately after the first train, the decline was hardly evident. If more than five minutes aftero the decline was particularly striking.

An interval of three minutes between stimulus trains was found to be the critical time, short ofwhich the fade in the second train was less than that in the conditioning train and, beyond which. the fade was greater. If trains of stimuli were delivered at three minute intervals, the degree of fade of the MAP responses remained similar on each occasion over periods of up to 30 minutes.

The conditioning train also affected the amplitude of the earliest potentials evoked during subsequent trains: when these were recorded immediately after the conditioning train, they were smaller than the first potentials of the conditioning train. Contrariwise, if the interval between stimulus trains exceeded three minutes, the first potentials of the second train were greater than those of the first. Voluntary contraction of the abductor digiti minimi had the same effect as the conditioning train, preventing the appearance of fade in the MAP responses evoked within a short time of contraction.

Similar changes were observed in the other patients studied (Fig. 1). Overall, the percentage decline of the MAP during the train was signi인

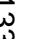


ficantly greater $(t=3 \cdot 11, P<0.05)$ when the study was performed after rest than when immediately after the previous train (Table). The fade did not always appear at the beginning of the train; in some instances, stimulation for a number of seconds was required (in patient M.C. a train of 20 seconds) before the fade was completed. The conditioning train also caused some depression of the first response of the second train when the interval between the two was short. The changes observed in each patient could be reproduced on subsequent days.

When stimulus trains of 50 second duration were used, the decline in amplitude of the MAP gave way to a subsequent increment which at times exceeded the initial response. This was most noticeable at a stimulus frequency of seven per second (Fig. 2), the changes occurring earlier at more rapid frequencies. Above 10 per second, however, this growth was only transient or absent. These changes were clearly evident in three of the four patients with myotonia congenita and in one patient with dystrophia myotonica.

In all patients, more rapid stimulus frequencies caused an earlier and more marked decline in the MAP (Fig. 3). The effect of a two second train of stimuli at 30 per second upon subsequent potentials evoked by one per second stimulation is shown in Fig. 4. Post-tetanic depression with subsequent recovery takes place.

EFFECT OF TEMPERATURE The effect of cooling and warming of the arm upon evoked MAP amplitude was studied in patient D.R. on two occasions. Eight second stimulus trains at 10 per second were delivered at three minute intervals for nine minutes. The arm and hand were then encased in a container of crushed ice and the stimulus trains continued at three minute intervals for 30 minutes by which time the surface temperature at the recording site had fallen to $28^{\circ} \mathrm{C}$. The ice was then removed and, after a further six minutes, the arm was warmed to $32^{\circ} \mathrm{C}$.

There was no change in the amplitude of the early MAPs in each train, though the degree of fade became slightly less 24 minutes after cooling had commenced. Within six minutes of the arm being warmed, the fade had returned to its previous value. Thus, cooling within this range does not exaggerate the MAP fade.

EFFECT OF EDROPHONIUM (TENSILON) The effect of $10 \mathrm{mg}$ edrophonium was observed in D.R. and G.H. (Fig. 5). In patient G.H., the fade of the evoked MAP became less striking, though the amplitude of the initial potential of each train of responses was unaltered. In patient D.R., this change was less obvious though, again, the degree of fade became less marked.

EFFECT OF GUANIDINE On two occasions in patient G.R., $800 \mathrm{mg}$ of guanidine hydrochloride was given intravenously over two minutes while eight second stimulus trains at 10 per second were delivered at three minute intervals. No significant change in either the amplitude or degree of fade of the evoked MAP responses was observed. On a separate occasion, the same dose of guanidine was given over two minutes while stimuli were delivered every second. Again, there was no change in the amplitude of the evoked MAP.

EFFECT OF SODIUM PHENYTOIN (EPANUTIN) Sodium phenytoin, $250 \mathrm{mg}$, was given intravenously over five minutes to patient G.R., while eight second stimulus trains at 10 per second were delivered at three minute intervals. No change in either the amplitude or degree of fade of the evoked MAP responses was observed.

DIRECT NERVE AND MUSCLE STIMULATION To find the site of the defect responsible for the fade in evoked MAP, the following studies of peripheral nerve and muscle were made in patient D.R. In the first, the amplitude of the mixed action potential of the ulnar nerve was recorded at the elbow through subcutaneous needle electrodes in response to stimulus trains at the wrist. No fade was observed during eight second stimulus trains of up to 40 per second: in particular, the amplitude did not change during stimulation at 10 per second whether the intervals between the stimulus trains were long or short (Fig. 6).

Next, using the technique described by McQuillen and Johns (1967) for the study of muscle excitability, trains of stimuli were delivered through a bipolar needle electrode 


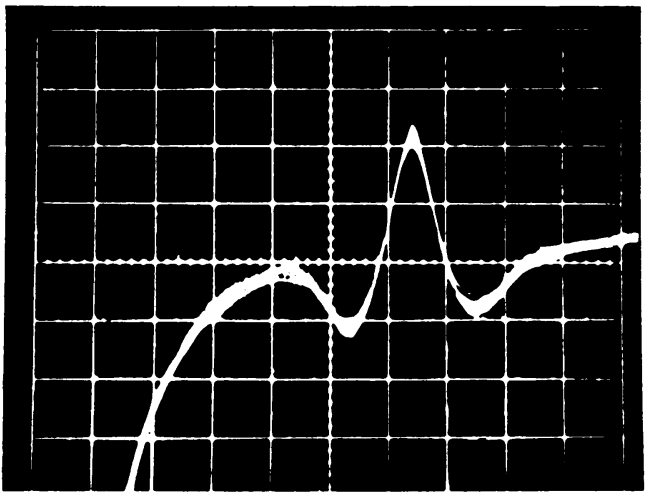

FIG. 6. Superimposed ulnar nerve action potentials recorded at the elbow evoked by an eight second stimulus train at 10 per second at the wrist in D.R. Vertical scale: $10 \mu \mathrm{V}$ per division. Horizontal scale: 1 ms per division.

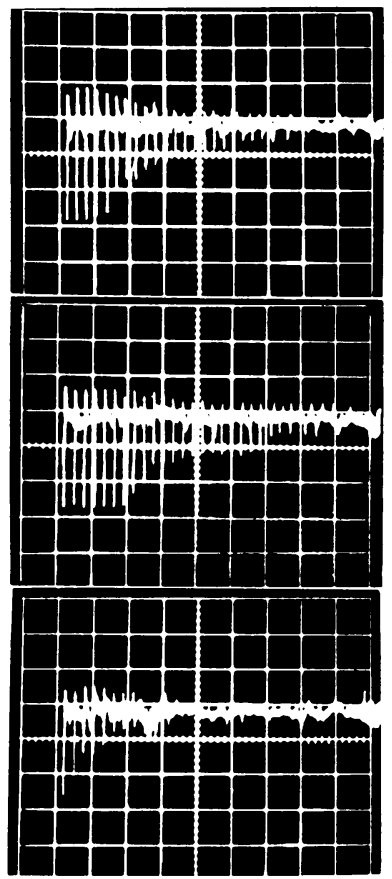

FIG. 7. MAP responses recorded from the left biceps evoked by three second trains of intramuscular electrical stimulation at seven per second in D.R. (see text). Vertical scale; $20 \mu \mathrm{V}$ per division. Horizontal scale; 0.5 seconds per division. Upper: three minutes after. Centre: I minute after. Lower: five minutes after the previous stimulus train. inserted in the biceps brachii muscle and the MAP recorded from concentric needle electrodes at a proximal site in the same muscle fibre. During 10 per second stimulus trains, there was a more rapid fade in the response than with neuromuscular stimulation. The effect of three second stimulus trains at seven per second was therefore observed (Fig. 7). It can be seen that this leads to the transient disappearance of the MAP. However, when the stimuli were delivered within 30 seconds of a previous train, the fade was less marked. Contrariwise, when the train was delivered after a five minute rest, the fade occurred earlier and was much more marked. Thus, the relationship between the degree of fade and the interval between the stimulus train and the previous conditioning train is similar to that which occurs with neuromuscular stimulation.

\section{DISCUSSION}

In normal subjects the amplitude of the evoked action potential of the abductor digiti minimi does not decline during 10 second stimulus trains of frequencies of up to 60 per second. Growth of the peak to peak amplitude at the commencement of the stimulus train indicates some degree of muscle shortening or better synchronization (seen in some of the patients with myotonia) but a subsequent fade does not occur (Simpson and Lenman, 1959). The present study confirms the findings of earlier workers with respect to the evoked MAP fade in myotonia. However, it is of particular interest that these changes appear to be so dependent upon whether the muscle is rested when the fade is most noticeable, or has recently contracted when the fade is less. This phenomenon bore little relationship to the degree of myotonia; in some instances it was very obvious where the clinical and electromyographic evidence of the myotonia was minimal: in others, it was not evident in muscles showing marked myotonia.

Questioning of patient D.R. revealed that his disability had two components which he clearly distinguished: the muscular stiffness and difficulty in relaxing his grip, most evident in the cold; and a tendency to transient weakness, more noticeable when he attempted sustained activity after rest. This latter feature must be related to the decremental evoked MAP res- 
ponses. Similar complaints of transient weakness were elicited from the other subjects with myotonia congenita.

The transient weakness and MAP fade in these patients should be distinguished from the periodic paralysis which may accompany paramyotonia congenita. Generalized attacks of weakness or paralysis did not occur, nor could localized weakness be induced by cold as in the latter condition (Magee, 1963, 1966). Furthermore, the evoked MAP fade occurred also in dystrophia myotonica in which condition periodic paralysis does not occur.

The dependence of this fade upon the timing of a previous conditioning stimulus train or voluntary contraction may explain the discrepancies among the earlier observations of this phenomenon. It may be overlooked if studies are not carried out on rested muscle or if short stimulus trains only are used. If the time between stimulus trains is not taken into account, then the erratic MAP responses which occur will prevent a proper study of the effect of drugs or temperature: hence, in D.R., the importance of defining the critical interval (three minutes) at which the fade during 10 per second stimulus trains remained the same, before such studies were undertaken.

The site of this defect must next be considered. A decremental MAP response is seen in myasthenic disorders: superficially, the 'warming up' feature of myotonia whereby the disability decreases during activity could be compared to the facilitation of strength during voluntary effort in the myasthenic syndrome of carcinoma (Eaton-Lambert syndrome), the result of a defect in the acetylcholine release mechanism (McQuillen and Johns, 1967). However, guanidine, which overcomes the defect in the latter condition, had no effect upon the MAP fade in myotonia. Furthermore, though edrophonium affected the degree of fade in two patients, sensitivity to curare is normal in myotonic disorders (Lambert and Beckett, 1950; Brown and Charlton, 1975), so there is no evidence for a defect of neuromuscular transmission. Since there is no fade in the amplitude of the action potential evoked in the ulnar nerve during repetitive stimulation, there is no evidence that defective nerve conduction could be responsible either. The fade is most striking when recordings are made from intramuscular electrodes in response to direct stimulation of muscle, indicating that an intrinsic change in the property of the myotonic muscle fibre must be responsible for this defect.

This can also be said for the site of the defect responsible for the hyper-excitability of the muscle fibre membrane which causes the repetitive depolarization of myotonic muscle (McComas and Johns, 1974). Could the same abnormality in myotonic muscle cause both the myotonia and the transient weakness reflected in the evoked MAP fade? The following points argue against this suggestion: patients can recognize that either 'stiffness or weakness' can be prominent at different times. Cooling, which increases the disability due to myotonia has very little effect on the evoked MAP fade and, finally, hydantoins, which improve the disability due to myotonia (Munsat, 1967), did not affect the evoked MAP fade.

In conclusion, in patients with myotonic disorders, it is usually possible to show significant fade of the evoked MAP. This indicates that a proportion of the muscle fibres cannot sustain their response to stimulation after being in the rested state, yet are capable of so doing for a short interval after activity. At the same time, immediately after a stimulus train, particularly at rapid rates, the amplitude of a single MAP response tends to be transiently depressed as illustrated by the post-tetanic depression in Fig. 4. Presumably, the interplay between these two distinct effects of a conditioning train upon the size of a subsequent evoked MAP is responsible for the fluctuating and varied responses and previous contradictory reports in myotonic disorders. These phenomena are due to altered function in the muscle fibre. Their cause is not yet clear.

The technical assistance of $\mathrm{Mr} \mathrm{P}$. Smith and the secretarial assistance of Miss $M$. Stevenson are acknowledged. Professor J. N. Walton and Dr D. A. Shaw are thanked for permission to study their patients. Illustrations were prepared in the Department of Medical Photography, United Norwich Hospitals. This work was supported by Grant 17912 from the Medical Research Council.

\section{REFERENCES}

Brown, J. C., and Charlton, J. E. (1975). A study of sensitivity to curare in certain neurological disorders using a regional 
technique. Journal of Neurology, Neurosurgery, and Psychiatry, 38. (In press.)

Grob, D., Johns, R. J., and Harvey, A. M. (1946). Studies in neuromuscular function. 1. Introduction and methods. Bulletin of the Johns Hopkins Hospital, 99, 115-124.

Kirby, J. F. Jr, and Kraft, G. H. (1973). Electromyographic studies in myotonia congenita. Archives of Physical Medicine and Rehabilitation, 54, 47-50, 68.

Lambert, E. J., and Beckett, S. (1950). Effect of epinephrine and other drugs on myotonia congenita. (Abstract) American Journal of Physiology, 163, 728.

McComas, A. J., Campbell, M. J., and Sica, R. E. P. (1971). Flectrophysiological study of dystrophia myotonica. Journal of Neurology, Neurosurgery, and Psychiatry, 34, 132-139.

McComas, A., and Johns, R. J. (1974). Potential changes in the normal and diseased muscle cell. In Disorders of Voluntary Muscle, 3rd edn, pp. 1073-1107. Edited by J. N. Walton. Churchill: London.

McQuillen, M. P., and Johns, R. J. (1967). The nature of the defect in the Eaton-Lambert syndrome. Neurology (Minneap.), 17, 527-536.

Magee, K. R. (1963). A study of paramyotonia congenita. Archives of Neurology, 8, 461-470.

Magee, K. R. (1966). Paramyotonia congenita. Association with cutaneous cold sensitivity and description of peculiar sustained postures after muscle contraction. Archives of Neurology, 14, 590-594.

Munsat, T. L. (1967). Therapy of myotonia. A double-blind evaluation of diphenylhydantoin, procainamide, and placebo. Neurology (Minneap.), 17, 359-367.

Ricker, K., Meinck, H.-M., and Stumpf, H. (1973). Neurophysiologische Untersuchungen über das Studium passagierer Lähmung bei Myotonia congenita und Dystrophia myotonica. Zeitschrift für Neurologie, 204, 135-148.

Simpson, J. A., and Lenman, J. A. R. (1959). The effect of frequency of stimulation in neuromuscular disease. (Abstract). Electroencephalography and Clinical Neurophysiology, 11, 604-605. 